\title{
Discovering the constrained NMSSM with tau leptons at the LHC
}

\author{
Ulrich Ellwanger, ${ }^{a}$ Alice Florent ${ }^{b}$ and Dirk Zerwas ${ }^{b}$ \\ ${ }^{a}$ Laboratoire de Physique Théorique, UMR 8627, CNRS and Université Paris-Sud 11, \\ Bât. 210, F-91405 Orsay, France \\ ${ }^{b}$ LAL, Université Paris-Sud 11, \\ CNRS/IN2P3, Orsay, France \\ E-mail: ellwanger@th.u-psud.fr, florent@lal.in2p3.fr, \\ zerwas@lal . in2p3.fr
}

AbStract: The constrained Next-to-Minimal Supersymmetric Standard Model (cNMSSM) with mSugra-like boundary conditions at the GUT scale implies a singlino-like LSP with a mass just a few GeV below a stau NLSP. Hence, most of the squark/gluino decay cascades contain two $\tau$ leptons. The gluino mass $\gtrsim 1.2 \mathrm{TeV}$ is somewhat larger than the squark masses of $\gtrsim 1 \mathrm{TeV}$. We simulate signal and background events for such a scenario at the LHC, and propose cuts on the transverse momenta of two jets, the missing transverse energy and the transverse momentum of a hadronically decaying $\tau$ lepton. This dedicated analysis allows to improve on the results of generic supersymmetry searches for a large part of the parameter space of the cNMSSM. The distribution of the effective mass and the signal rate provide sensitivity to distinguish the cNMSSM from the constrained Minimal Supersymmetric Standard Model in the stau-coannihilation region.

KeYWORDs: Supersymmetry Phenomenology

ARXIV EPRINT: 1011.0931 


\section{Contents}

1 Introduction $\quad 1$

2 The spectrum of the cNMSSM 3

$\begin{array}{lll}3 & \text { The signal, backgrounds and cuts } & 6\end{array}$

4 Simulation, signal and background rates $\quad 7$

5 Comparison with the cMSSM in the stau coannihilation region 10

6 Conclusions and outlook $\quad 14$

\section{Introduction}

The Next-to-Minimal Supersymmetric Standard Model (NMSSM, for recent reviews see [1, 2]) provides an elegant solution to the $\mu$-problem of the Minimal Supersymmetric Standard Model (MSSM) [3]: after the replacement of the $\mu$-term in the superpotential of the MSSM by the coupling to a gauge singlet superfield $S$, the superpotential is scale invariant and the only dimensionful parameters in the Lagrangian are soft Supersymmetry (SUSY) breaking terms.

After electroweak symmetry breaking, all components of the gauge singlet superfield $S$ mix with the components of the MSSM-like Higgs doublet superfields $H_{u}$ and $H_{d}$. Accordingly NMSSM specific phenomena can take place in the CP-even Higgs sector, the CP-odd Higgs sector and the neutralino sector. In this paper we concentrate on the neutralino sector, where the 5th singlet-like neutralino (in addition to the two neutral higgsinos, the neutral wino and the bino) can be the Lightest Supersymmetric Particle (LSP) [4-8] and, simultanously, give rise to a dark matter density in agreement with WMAP constraints [9-14].

Such a scenario is not far fetched: A conceptually simple origin of soft SUSY breaking terms is a minimal coupling to supergravity (with a flavour independent Kähler potential and minimal gauge kinetic terms), and the assumption of spontaneously broken SUSY in a hidden sector. Then, the soft SUSY breaking terms are universal at the Planck scale (not far from the GUT scale) in the form of universal scalar masses $m_{0}$, universal gaugino masses $M_{1 / 2}$ and universal trilinear scalar couplings proportional to $A_{0}$.

The correspondingly constrained NMSSM (cNMSSM) has been studied first in [15-20]. Since then, the precision of the radiative corrections has been considerably improved, and the computation of the dark matter relic density has become possible [9]. Imposing the requirement of a dark matter relic density in agreement with WMAP constraints [14] as well as present constraints on Higgs and supersymmetric particle (sparticle) masses, the parameter space of the cNMSSM has been analysed recently in [21, 22]. (For studies of 
the semi-constrained NMSSM, where the singlet-dependent SUSY breaking parameters are allowed to be non-universal, see [12, 13, 23-25].)

Within the cNMSSM, the dark matter constraints require a singlino-like LSP. The origin of this phenomenon is quite easy to understand: first, the CP-even scalar singlet $s$ has to assume a non-vanishing vacuum expectation value (vev) in order to generate the required $\mu$-term. For this reason its SUSY breaking mass squared $m_{S}^{2}$ at the electroweak scale must not be very large; otherwise the minimum of its potential is at $s=0$. Second, $m_{S}^{2}$ is hardly renormalized between the GUT and the electroweak scales, which leads to $m_{S}^{2} \sim m_{0}^{2}$ with the consequence that, in the cNMSSM, $m_{0}$ must be small compared to $M_{1 / 2}$ and $A_{0}$.

It is well known that, within the cMSSM [26], a small value of $m_{0}$ would imply a stau $\left(\widetilde{\tau}_{1}\right)$ LSP, which is not a reasonable candidate for the dark matter. In the cNMSSM, the singlino-like neutralino $\chi_{1}^{0}$ can — and must - be lighter than the $\widetilde{\tau}_{1}$ for this reason. In order for its dark matter relic density not being too large, its mass must be only a few $\mathrm{GeV}$ below the mass of the $\widetilde{\tau}_{1}$ such that $\chi_{1}^{0}-\widetilde{\tau}_{1}$ coannihilation processes are sufficiently fast $[12,21,22]$.

Such a scenario would necessarily have a strong impact on sparticle searches at colliders (see $[27,28]$ for reviews of searches at the LHC): since the $\widetilde{\tau}_{1}$ is the NLSP and the singlinolike neutralino $\chi_{1}^{0}$ couples only very weakly to all sparticles, the sparticles decay first into the $\widetilde{\tau}_{1}$ under the emission of at least one $\tau$-lepton. Subsequently the $\widetilde{\tau}_{1}$ decays into $\chi_{1}^{0}+\tau$, hence each sparticle decay cascade contains typically two $\tau$-leptons. In the present paper we study, for the first time, the corresponding implications for sparticle searches at the LHC.

As discussed in [21, 22], the sparticle (and Higgs) spectrum is quite constrained in the cNMSSM, and can essentially be parametrized by $M_{1 / 2}$. The present lower bounds on $m_{\widetilde{\tau}_{1}}$ and the lower LEP bound of $\sim 114 \mathrm{GeV}$ on the CP-even Standard Model like Higgs mass require $M_{1 / 2} \gtrsim 520 \mathrm{GeV}$ which, inspite of $m_{0} \lesssim 50 \mathrm{GeV}$, implies a quite heavy sparticle spectrum: squark masses $\gtrsim 1 \mathrm{TeV}$ (apart from the somewhat lighter stop and sbottom masses), and a gluino mass $\gtrsim 1.2 \mathrm{TeV}$. Since consequently squark production will be relatively frequent compared to gluino production (in fact, squark + gluino production dominates), the signal events will mostly lead to two jets (not counting ISR and FSR) with a quite large $p_{\mathrm{T}}$ as well as a large missing transverse energy $E_{\mathrm{T}}^{\text {miss }}$. The sum of the squark and/or gluino production cross sections is, however, just $\sim 1 \mathrm{pb}$ or less (for larger $M_{1 / 2}$ ).

Each sparticle production event in the cNMSSM will contain typically four $\tau$-leptons in the final state: two of the $\tau$-leptons (those originating from $\widetilde{\tau}_{1}$ decays into $\chi_{1}^{0}+\tau$ ) will be quite soft due to the small $\widetilde{\tau}_{1}-\chi_{1}^{0}$ mass difference $\lesssim 5 \mathrm{GeV}$ and are difficult to detect, whereas the $\tau$-leptons from decays as $\chi_{2}^{0} \rightarrow \widetilde{\tau}_{1}+\tau$ are relatively energetic.

The aim of the present paper is to show that a dedicated analysis allows for much better signal to background ratios for the cNMSSM than standard (generic) supersymmetric analyses. We simulate and study signals and various Standard Model backgrounds for the LHC at $14 \mathrm{TeV}$ c.m. energy, and find that the signal to background ratio is sufficiently large allowing for the discovery of the cNMSSM for a wide range of values of $M_{1 / 2}$.

Events with $E_{\mathrm{T}}^{\text {miss }}$, jets and $\tau$-leptons could also be a signal for the (c)MSSM in the so-called stau-coannihilation region [29-33]. Since the squark/neutralino spectrum of the 
cMSSM is necessarily different from the cNMSSM, the combination of $M_{\text {eff }}$ (essentially the sum of the transverse momenta and $E_{\mathrm{T}}^{\text {miss }}$ ) and the signal rate has sensitivity to distinguish the two models.

In the next section we present the spectrum of the cNMSSM. In section 3 we discuss the signal, backgrounds and appropriate cuts. Details of the simulation of the cNMSSM signal and the Standard Model backgrounds, the effect of cuts, and the resulting cross sections and signal to background ratios are given in section 4 . Section 5 is devoted to the comparison of the cNMSSM with the cMSSM, and section 6 to conclusions and an outlook.

\section{The spectrum of the cNMSSM}

The NMSSM with a scale invariant superpotential $W[1,2]$ differs from the MSSM through the replacement of the $\mu$ term in $W_{M S S M}$ by the coupling to a gauge singlet superfield $S$ and a trilinear $S$ self-coupling:

$$
W_{M S S M}=\mu H_{u} H_{d}+\ldots \rightarrow W_{N M S S M}=\lambda S H_{u} H_{d}+\frac{\kappa}{3} S^{3}+\ldots,
$$

where we have omitted the quark/lepton Yukawa couplings. Hence, if the vev $s$ of $S$ is non-zero (induced by the soft SUSY breaking terms), an effective $\mu$ term $\mu_{\text {eff }}=\lambda s$ of the desired order of magnitude is generated and solves the $\mu$ problem of the MSSM [3].

Apart from the Yukawa couplings in the superpotential and the gauge couplings, the Lagrangian of the NMSSM depends on soft SUSY breaking scalar masses for the Higgs fields $H_{u}, H_{d}$ and $S$, the squarks and the sleptons; trilinear couplings among the scalars (proportional to the couplings in the superpotential); and gaugino masses for the bino $\left(M_{1}\right)$, the winos $\left(M_{2}\right)$ and the gluino $\left(M_{3}\right)$. Assuming supersymmetry breaking from a hidden sector in minimal supergravity (mSUGRA), the SUSY breaking terms are assumed to be universal at the scale of grand unification (near the Planck scale) and denoted as $m_{0}, A_{0}$ and $M_{1 / 2}$, respectively. Hence the parameters of the corresponding cNMSSM are, apart from the gauge and quark/lepton Yukawa couplings,

$$
m_{0}, A_{0}, M_{1 / 2}, \lambda \text { and } \kappa \text {. }
$$

It is convenient to fix $\kappa$ from the requirement that the Higgs vevs $h_{u}$ and $h_{d}$ generate the correct value of $M_{Z}$.

As mentioned in the introduction and discussed in detail in [21, 22], the remaining parameters in (2.2) are strongly constrained: $m_{0}$ must be small such that the vev $s$ is non-zero. A small non-zero value for $m_{0}$ affects essentially only the singlet-like CP-even Higgs mass [22], which is irrelevant for the present study; hence we assume $m_{0}=0$ in the following. In order to avoid a $\widetilde{\tau}_{1}$ LSP, the singlino (the fermionic component of $S$ ) must be lighter such that $\widetilde{\tau}_{1}$ is the NLSP. The singlino relic density can be reduced to an amount compatible with WMAP constraints, if its co-annihilation rate with the $\widetilde{\tau}_{1}$ is large enough, i.e. if the corresponding mass difference is sufficiently small. This fixes $A_{0} \sim-\frac{1}{4} M_{1 / 2}[21,22]$. Finally $\lambda$ must also be quite small, since $\lambda$ induces mixings in the CP-even Higgs sector between the doublet- and singlet-like Higgs states whose masses 
are always of the same order of magnitude in the cNMSSM. If the singlet-like Higgs state is slightly heavier than the Standard Model like Higgs state $h$, the mixing decreases the mass of $h$. Then its mass falls below the LEP bound of $\sim 114 \mathrm{GeV}$ if $\lambda$ is too large. If the mass of singlet-like Higgs state is below $m_{h}$ (below $114 \mathrm{GeV}$ as for the point P520 in table 1 below), its coupling to the $Z$ boson violates again LEP bounds [34] for $\lambda$ too large. All in all one finds $\lambda \lesssim 0.02[21,22]$ (but $\lambda \gtrsim 10^{-5}$ in order still to allow for singlino- $\widetilde{\tau}_{1}$ co-annihilation).

$\lambda$ induces also mixings between the singlet-like neutralino and the MSSM-like neutralinos (bino, neutral wino and higgsinos). For $\lambda \lesssim 0.02$, these mixings are very small. Hence the couplings of the singlino-like LSP $\chi_{1}^{0}$ to all MSSM-like sparticles (squarks, gluino, sleptons, charginos and neutralinos), which are induced by these mixings, are very small as well. Accordingly branching ratios of all these sparticles into the singlino-like LSP are negligibly small, unless a decay into $\chi_{1}^{0}$ is the only decay possible. Due to R-parity conservation this is the case for the NLSP, the $\widetilde{\tau}_{1}$. Hence sparticle decay cascades proceed as in the MSSM (with a spectrum as in the cNMSSM, but without the singlet-like states) until the $\widetilde{\tau}_{1}$ NLSP is produced. Depending on $\lambda$, the width of the final decay $\widetilde{\tau}_{1} \rightarrow \chi_{1}^{0}+\tau$ can be so small, that the $\widetilde{\tau}_{1}$ decay vertex is visibly displaced [22]. This case (where the displaced vertex corresponds to the production of a soft $\tau$-lepton) could be another interesting signature for the cNMSSM, but subsequently we will not assume that $\lambda$ is so small that this phenomenon occurs.

Concerning the remaining parameter $M_{1 / 2}$, we find that the LEP constraints on the Higgs sector require $M_{1 / 2} \gtrsim 520 \mathrm{GeV}$. Then, all bounds on sparticle masses from colliders as well as constraints from B-physics are satisfied. For the calculation of the spectrum we use the code NMSPEC [23] within NMSSMTools [35, 36], updated including radiative corrections to the Higgs sector from [37]. The dark matter relic density is computed with the help of micrOMEGAs [9]. Clearly, very large values of $M_{1 / 2}$ are generally disfavoured by fine-tuning arguments; moreover, smaller values of $M_{1 / 2} \lesssim 1 \mathrm{TeV}$ allow to explain the discrepancy of the measured anomalous magnetic moment of the muon with the Standard Model [22]. Subsequently we confine ourselves to $M_{1 / 2} \leq 1 \mathrm{TeV}$. In table 1 we show the values for $A_{0}, \tan \beta=h_{u} / h_{d}$ and $\mu_{\text {eff }}$ ( $A_{0}$ is determined by the correct dark matter relic density, whereas $\tan \beta$ and $\mu_{\text {eff }}$ are obtained as output) as well as the Higgs and sparticle spectra for $M_{1 / 2}=520,600,800 \mathrm{GeV}$ and $1 \mathrm{TeV}$ for $m_{0}=0$ and $\lambda=0.001$.

We see that, as announced above, $m_{\widetilde{\tau}_{1}}-m_{\chi_{1}^{0}} \lesssim 5 \mathrm{GeV}$ and hence $\tau$-leptons from the decay $\widetilde{\tau}_{1} \rightarrow \chi_{1}^{0}+\tau$ are necessarily soft. $\tau$-leptons from the decay $\chi_{2}^{0} \rightarrow \widetilde{\tau}_{1}+\tau$ (where $\chi_{2}^{0}$ is dominantly bino-like) profit at least from $m_{\chi_{2}^{0}}-m_{\widetilde{\tau}_{1}} \sim 70 \mathrm{GeV}$ (for P520), or more energy from the decays of other sparticles into $\widetilde{\tau}_{1}$. Note that right-handed sleptons $\widetilde{e}_{R}$ and $\widetilde{\mu}_{R}$ decay essentially via the three-body channel as $\widetilde{e}_{R} \rightarrow e+\widetilde{\tau}_{1}+\tau$. In fact, apart from the NMSSM-specific decay $\widetilde{\tau}_{1} \rightarrow \chi_{1}^{0}+\tau$ (with a branching ratio of $100 \%$ ), the corresponding sparticle decay branching ratios can be obtained from the code SUSY-HIT [38] and the MSSM with a corresponding spectrum, which is used for the simulations of events below.

At the LHC, the dominant sparticle production processes are of course squark-gluino and squark-(anti-)squark pair productions. Subsequently a typical squark decay cascade looks like

$$
\widetilde{q} \rightarrow q+\chi_{2}^{0} \rightarrow q+\tau+\widetilde{\tau_{1}} \rightarrow q+\tau+\tau+\chi_{1}^{0}
$$




\begin{tabular}{|l|c|c|c|c|}
\hline & P520 & P600 & P800 & P1000 \\
\hline$M_{1 / 2}(\mathrm{GeV})$ & 520 & 600 & 800 & 1000 \\
\hline$A_{0}(\mathrm{GeV})$ & -142 & -166 & -225 & -282 \\
\hline $\tan \beta$ & 23.2 & 24.3 & 26.6 & 28.3 \\
\hline$\mu_{\mathrm{eff}}(\mathrm{GeV})$ & 666 & 757 & 977 & 1190 \\
\hline$m_{h_{1}^{0}}(\mathrm{GeV})$ & 100 & 115 & 117 & 118 \\
\hline$m_{h_{2}^{0}}(\mathrm{GeV})$ & 115 & 118 & 159 & 199 \\
\hline$m_{h_{3}^{0}}(\mathrm{GeV})$ & 654 & 738 & 937 & 1127 \\
\hline$m_{a_{1}^{0}}(\mathrm{GeV})$ & 174 & 203 & 275 & 345 \\
\hline$m_{a_{2}^{0}}(\mathrm{GeV})$ & 654 & 738 & 937 & 1127 \\
\hline$m_{h^{ \pm}}(\mathrm{GeV})$ & 667 & 751 & 951 & 1140 \\
\hline$m_{\chi_{1}^{0}}(\mathrm{GeV})$ & 142 & 166 & 225 & 282 \\
\hline$m_{\chi_{2}^{0}}(\mathrm{GeV})$ & 215 & 250 & 338 & 427 \\
\hline$m_{\chi_{3}^{0}}(\mathrm{GeV})$ & 404 & 471 & 636 & 801 \\
\hline$m_{\chi_{4,5}^{0}}(\mathrm{GeV})$ & 680 & 770 & 990 & 1200 \\
\hline$m_{\chi_{1}^{ \pm}}(\mathrm{GeV})$ & 404 & 471 & 636 & 801 \\
\hline$m_{\chi_{2}^{ \pm}}(\mathrm{GeV})$ & 684 & 773 & 992 & 1203 \\
\hline$m_{\tilde{g}}(\mathrm{GeV})$ & 1192 & 1361 & 1777 & 2187 \\
\hline$m_{\tilde{u}_{L}}(\mathrm{GeV})$ & 1082 & 1234 & 1607 & 1973 \\
\hline$m_{\tilde{u}_{R}}(\mathrm{GeV})$ & 1044 & 1189 & 1546 & 1895 \\
\hline$m_{\tilde{d}_{L}}(\mathrm{GeV})$ & 1085 & 1237 & 1609 & 1974 \\
\hline$m_{\tilde{d}_{R}}(\mathrm{GeV})$ & 1040 & 1184 & 1539 & 1886 \\
\hline$m_{\tilde{t}_{1}}(\mathrm{GeV})$ & 825 & 947 & 1246 & 1538 \\
\hline$m_{\tilde{t}_{2}}(\mathrm{GeV})$ & 1032 & 1165 & 1492 & 1816 \\
\hline$m_{\tilde{b}_{1}}(\mathrm{GeV})$ & 973 & 1109 & 1444 & 1772 \\
\hline$m_{\tilde{b}_{2}}(\mathrm{GeV})$ & 1020 & 1158 & 1496 & 1826 \\
\hline$m_{\tilde{e}_{L}}(\mathrm{GeV})$ & 347 & 399 & 527 & 654 \\
\hline$m_{\tilde{e}_{R}}(\mathrm{GeV})$ & 196 & 224 & 296 & 368 \\
\hline$m_{\tilde{\nu}_{l}}(\mathrm{GeV})$ & 338 & 391 & 521 & 650 \\
\hline$m_{\tilde{\tau}_{1}}(\mathrm{GeV})$ & 147 & 171 & 229 & 286 \\
\hline$m_{\tilde{\tau}_{2}}(\mathrm{GeV})$ & 353 & 403 & 525 & 647 \\
\hline$m_{\tilde{\nu}_{\tau}}(\mathrm{GeV})$ & 332 & 383 & 509 & 633 \\
\hline$\sigma$ (pb & 1.36 & 0.70 & 0.134 & 0.035 \\
\hline
\end{tabular}

Table 1. Input parameters, $\tan \beta, \mu_{\mathrm{eff}}$ and low-energy spectra for four points of the cNMSSM with $m_{0}=0$ and $\lambda=0.001$. In the last line we give the total NLO cross sections for the production of all sparticles at the LHC. 
but many more possibilities exist. Their simulation, together with the simulation of Standard Model background processes, will be discussed in the next sections.

\section{The signal, backgrounds and cuts}

As for most SUSY models, the production of squarks of the first generation and of gluinos will be the dominant sparticle production processes at the LHC. Their total production cross sections are obtained at NLO (QCD) from PROSPINO [39], and are also shown in table 1. The dominant contributions originate from squark + gluino production $(\sim 37 \%)$ and squark pair production $(\sim 25 \%)$; less dominant are squark + antisquark production $(\sim 13 \%)$ and gluino pair production $(\sim 5 \%)$. The production cross sections of stop and sbottom squarks, sleptons, charginos and neutralinos add another $\sim 19 \%$ to the total sparticle production cross sections.

The dominant background processes for SUSY searches are well-known: top-antitop pair production, $\mathrm{W}+\mathrm{n}$-jet production, $\mathrm{Z}+\mathrm{n}$-jet production, $\mathrm{W}+\mathrm{Z}$ production and $\mathrm{WW}+\mathrm{n}-$ jet production. Since we will compare the performance of our simulation with the results of standard SUSY searches by ATLAS [40], we assume the same production cross sections for these background processes as in [40] (given in table 2 below).

Given that gluinos (whose decay generates typically two hard jets) are even somewhat heavier than the first generation squarks (generating typically one hard jet), we require at least two hard jets per event only whereas in generic supersymmetric analyses usually four hard jets are required. On the other hand, given the large squark and gluino masses, we can require quite large transverse momenta of the jets as well as a large missing transverse energy $E_{\mathrm{T}}^{\mathrm{miss}}$.

For the $\tau$-leptons we consider their hadronic decays only. For their transverse momenta we require at least $30 \mathrm{GeV}$, which allows to assume an efficiency of $\sim 40 \%$ [40] (and a $\tau$-fake rate of jets of $\sim 1-2 \%$ ). On the other hand, since only two among the four $\tau$-leptons per event are sufficiently energetic and the total signal cross sections are already quite small, we require one identified $\tau$-lepton only.

Additional standard cuts are a lower limit on the angle $\Delta \Phi$ between the hard jets and $E_{\mathrm{T}}^{\mathrm{miss}}$, as well as a cut on the transverse mass $M_{T}$ formed from $E_{\mathrm{T}}^{\mathrm{miss}}$ and the identified $\tau$-lepton (in order remove semileptonically decaying $\mathrm{W}+$ jets events). Altogether, the list of our cuts is given by:

1. At least two jets, one with $p_{\mathrm{T}}>300 \mathrm{GeV}$ and one with $p_{\mathrm{T}}>150 \mathrm{GeV}$

2. $E_{\mathrm{T}}^{\text {miss }}>300 \mathrm{GeV}$

3. At least one $\tau$-lepton with $p_{\mathrm{T}}>30 \mathrm{GeV}$

4. $\Delta \Phi\left(j_{i}, E_{\mathrm{T}}^{\mathrm{miss}}\right)>0.2$ for the hard jets

5. $M_{T}>100 \mathrm{GeV}$, where $M_{T}$ is computed from the visible momenta of the hardest $\tau$-lepton and $E_{\mathrm{T}}^{\text {miss }}$.

Below we will denote this set of cuts as cNMSSM analysis. 


\section{Simulation, signal and background rates}

Both the signal and the top quark background were generated by PYTHIA 6.4 [41], which was in charge of generation and phase-space decays. PYTHIA performed the parton showering as well as the matching procedure according to the MLM prescription including initial and final state radiation. TAUOLA [43-45] was employed for the $\tau$-decays. All other backgrounds (involving at least one $\mathrm{W}$ or Z-boson) were generated with ALPGEN [42]. In order to keep the statistics manageable, preselection cuts were applied on the ALPGEN samples. Since the ALPGEN multijet generation is very (CPU)-time consuming, we had to limit the SM sample to about $1 \mathrm{fb}^{-1}$. Since at least 120000 events were generated per point for the signal, the statistical fluctuations of the SM background (after cuts) will be consideraby larger.

Since event generation was performed by leading order generators, the cross sections were scaled according to the NLO cross sections in table 1 for the signal, as in [40] to the NLO+NLL calculation for top production, and to NLO (or NNLO level, where available) for electroweak boson(s) production.

For the detector simulation we employed AcerDet [46]. AcerDet is a fast detector simulation which provides a reasonable description of the performance of an LHC detector. The events generated by PYTHIA and ALPGEN+PYTHIA were all passed through AcerDet. AcerDet reconstructs jets, leptons and the missing transverse energy, it also labels the origin of the jets, e.g., those coming from a tau lepton. The efficiency and the corresponding background rejection for a working point of $40 \% \tau$ identification efficiency were implemented at reconstruction level.

One of the issues to be checked is the energy of the reconstructed tau-jets. For this initial check we used PYTHIA to produce Z bosons and their subsequent decay to tau leptons. The hadron-hadron as well as the lepton-hadron final states were reconstructed and compared to the results of the ATLAS collaboration presented in [40]. Reasonable agreement at the level of a few percent (about $3 \mathrm{GeV}$ ) was found.

A fast detector simulation as AcerDet will not be able to simulate the non-Gaussian tails, e.g., in the transverse missing energy distribution. Simulation and reconstruction results without full detector simulation and reconstruction are therefore not expected to be perfectly reproduced. To get a feeling of how well the background can be modeled with AcerDet, two signatures of ref. [40] have been implemented and analysed in addition to the dedicated cNMSSM analysis: the four-jet SUSY search (4j0l) and the SUSY search with at least one tau in the final state (4jtau).

In table 2 the result of the background cross sections before and after the cuts is shown for the two ATLAS as well as the cNMSSM signatures. For the $4 \mathrm{j} 01$ analyses, the AcerDet-result for the total background cross section of about $606 \mathrm{fb}$ after cuts is of the right order of magnitude compared to $708 \mathrm{fb}$ (NLO) in [40]. Adding the tau identification to the analysis in the 4 jtau analysis, a total background cross section of $61 \mathrm{fb}$ after cuts obtained here is to be compared with $51 \mathrm{fb}$ at NLO in [40]. Thus with and without tau identification, AcerDet provides a reasonable estimate of the expected background with respect to the dedicated full simulation. 


\begin{tabular}{|c|cc|c|c|c|}
\hline & Events & cross section $(\mathrm{pb})$ & 4 j0l $(\mathrm{fb})$ & 4jtau $(\mathrm{fb})$ & cNMSSM $(\mathrm{fb})$ \\
\hline tt & 2110000 & 833 & $350 \pm 12$ & $50 \pm 4.4$ & $7.9 \pm 1.7$ \\
\hline W+2jets & 222700 & 281 & 0 & 0 & 0 \\
W+3jets & 89250 & 116 & $15 \pm 4.5$ & 0 & $2.6 \pm 1.8$ \\
W+4jets(inc) & 50875 & 61 & $220 \pm 16$ & $9.6 \pm 3.4$ & $4.8 \pm 2.4$ \\
\hline W+jets (inc) & & & $235 \pm 16.6$ & $9.6 \pm 3.4$ & $7.4 \pm 3$ \\
\hline Z+2jets & 88850 & 106 & 0 & 0 & 0 \\
Z+3jets & 22320 & 27.5 & 0 & 0 & 0 \\
Z+4jets(inc) & 11639 & 10.1 & $0.9 \pm 0.9$ & 0 & 0 \\
\hline Z+jets & & & $0.9 \pm 0.9$ & 0 & 0 \\
\hline ZW(inc) & 250 & 0.5 & $2 \pm 2$ & 0 & 0 \\
\hline WW+0jet & 50000 & 47 & 0 & 0 & 0 \\
WW+1jet & 15000 & 20 & 0 & 0 & 0 \\
WW+2jet(inc) & 32796 & 13 & $17.8 \pm 2.6$ & $1.2 \pm 0.7$ & $0.8 \pm 0.6$ \\
\hline WW+jets (inc) & & & $17.8 \pm 2.6$ & $1.2 \pm 0.7$ & $0.8 \pm 0.6$ \\
\hline Total & & $606 \pm 21$ & $61 \pm 5.6$ & $16.1 \pm 3.5$ \\
\hline
\end{tabular}

Table 2. The number of simulated background events, cross sections before cuts, and cross sections after the 4j0l, $4 \mathrm{jtau}$ and the cNMSSM analysis. The quoted error is the statistical error.

We note that already with the softer cuts within the $4 \mathrm{j} 01$ analyses and notably the cuts on $\Delta \Phi\left(j_{i}, E_{\mathrm{T}}^{\mathrm{miss}}\right)$, the remaining QCD background was found to be small in [40]. In our case, QCD events could pass the cNMSSM cuts only if a very large value of $E_{\mathrm{T}}^{\mathrm{miss}}$ and a $\tau$-lepton would be faked simultaneously. Assuming a jet $\rightarrow \tau$ fake rate up to $\sim 2 \%$ (for an acceptance of $40 \%$ ), and that the suppression rate of QCD events without missing energy for $\left.E_{\mathrm{T}}^{\mathrm{miss}}\right)>150 \mathrm{GeV}$ is $1 \%$ [40] while we cut at $300 \mathrm{GeV}$, we should be safe of the QCD background.

Whereas the two ATLAS analyses are designed to cover a large variety of supersymmetric signals, the signatures discussed above are chosen specifically for heavy squarks and gluinos as well as $\tau$-rich final states as in the cNMSSM. The background cross sections for this analysis are shown in the last column of table 2. The total background, already decreased from the 4-jet-0-lepton to the 4-jet-tau analysis by an order of magnitude, is reduced by another factor four to $\sim 16 \mathrm{fb}$.

Typically the overall efficiency for all SUSY processes weighted by the cross section varies between $7 \%$ and $10 \%$. The cross section for the cNMSSM benchmark points after all cuts are shown in table 3 for the three analyses. The $S / B$ ratio, the ratio $S / \sqrt{B}$ for an integrated luminosity of $1 \mathrm{fb}^{-1}$ as well as for $30 \mathrm{fb}^{-1}$ are shown.

Table 3 clarifies the advantage of the cNMSSM cuts with respect to the general analysis: the ratio $S / \sqrt{B}$ is $7-10$ times larger for the cNMSSM cuts even with respect to the standard 4-jet-tau signature, which originates both from the larger background suppression and the larger efficiency on the signal. Correspondingly the cNMSSM cuts allow for a sensitivity, for a given luminosity, on a much larger part of the cNMSSM parameter space (for heavier squarks/gluinos). The point P800 (with squark/gluino masses of $1.6 / 1.7 \mathrm{TeV}$ ) is hardly 


\begin{tabular}{|c|c|c|c|c|}
\hline & & $4 \mathrm{j} 0 \mathrm{l}$ & $4 \mathrm{jtau}$ & cNMSSM \\
\hline $\mathrm{P} 520$ & $1.36 \mathrm{pb}$ & $101 \pm 1.1 \mathrm{fb}$ & $27 \pm 0.5 \mathrm{fb}$ & $99 \pm 1 \mathrm{fb}$ \\
$\mathrm{S} / \mathrm{B}$ & & 0.17 & 0.44 & 6.2 \\
$\mathrm{~S} / \sqrt{\mathrm{B}}$ & $1 \mathrm{fb}^{-1}$ & 4.1 & 3.4 & 24.8 \\
$\mathrm{~S} / \sqrt{\mathrm{B}}$ & $30 \mathrm{fb}^{-1}$ & 22 & 19 & 136 \\
\hline $\mathrm{P} 600$ & $0.70 \mathrm{pb}$ & $47 \pm 0.5 \mathrm{fb}$ & $13.3 \pm 0.3 \mathrm{fb}$ & $58 \pm 0.6 \mathrm{fb}$ \\
$\mathrm{S} / \mathrm{B}$ & & 0.08 & 0.21 & 3.6 \\
$\mathrm{~S} / \sqrt{\mathrm{B}}$ & $1 \mathrm{fb}^{-1}$ & 1.9 & 1.7 & 14.5 \\
$\mathrm{~S} / \sqrt{\mathrm{B}}$ & $30 \mathrm{fb}^{-1}$ & 10.5 & 9.3 & 79 \\
\hline $\mathrm{P} 800$ & $0.134 \mathrm{pb}$ & $7.5 \pm 0.1 \mathrm{fb}$ & $2.7 \pm 0.05 \mathrm{fb}$ & $13.4 \pm 0.1 \mathrm{fb}$ \\
$\mathrm{S} / \mathrm{B}$ & & 0.012 & 0.04 & 0.8 \\
$\mathrm{~S} / \sqrt{\mathrm{B}}$ & $1 \mathrm{fb}^{-1}$ & 0.3 & 0.34 & 3.4 \\
$\mathrm{~S} / \sqrt{\mathrm{B}}$ & $30 \mathrm{fb}^{-1}$ & 1.7 & 1.9 & 18 \\
\hline $\mathrm{P} 1000$ & $0.035 \mathrm{pb}$ & $1.68 \pm 0.02 \mathrm{fb}$ & $0.62 \pm 0.01 \mathrm{fb}$ & $3.43 \pm 0.03 \mathrm{fb}$ \\
$\mathrm{S} / \mathrm{B}$ & & 0.002 & 0.01 & 0.2 \\
$\mathrm{~S} / \sqrt{\mathrm{B}}$ & $1 \mathrm{fb}^{-1}$ & 0.07 & 0.07 & 0.86 \\
$\mathrm{~S} / \sqrt{\mathrm{B}}$ & $30 \mathrm{fb}^{-1}$ & 0.37 & 0.43 & 4.7 \\
\hline
\end{tabular}

Table 3. Signal expectation for the NMSSM points at NLO after all cuts for the benchmark points. At least 120000 events per point were generated. The error is the statistical error. For every point the ratios $\mathrm{S} / \mathrm{B}$ and $\mathrm{S} / \sqrt{\mathrm{B}}$ for an integrated luminosity of $1 \mathrm{fb}^{-1}$ and $30 \mathrm{fb}^{-1}$ are shown.

visible within the standard analysis even for $30 \mathrm{fb}^{-1}$, whereas the ratio $\mathrm{S} / \sqrt{\mathrm{B}}$ is still $\sim 18$ for the cNMSSM cuts. Only for the point P1000 (with squark/gluino masses of 1.9/2.2 TeV) a larger luminosity and/or even harder cuts seem to be required for detection.

In figure 1 we show the spectrum of the effective mass $M_{\mathrm{eff}} \equiv \sum p_{\mathrm{T}}^{\mathrm{jets}}+\sum p_{\mathrm{T}}^{\text {lep }}+E_{\mathrm{T}}^{\mathrm{miss}}$ after all cuts of the cNMSSM analysis are applied, normalised to an integrated luminosity of $1 \mathrm{fb}^{-1}$. Typically, the spectrum of the effective mass peaks at a value corresponding to the masses of the pair produced sparticles [40]. Here the maxima of $M_{\text {eff }}$ are shifted to somewhat larger values due to the cuts on $p_{\mathrm{T}}^{\text {jets }}$ and $E_{\mathrm{T}}^{\text {miss }}$.

For the SM background the average effective mass is $\left\langle M_{\text {eff }}(S M)\right\rangle=1600 \mathrm{GeV}$. The error on the estimation of the effective mass is $120 \mathrm{GeV}$ for $1 \mathrm{fb}^{-1}$, including both the statistical error of the (future) data sample as well as the uncertainty due to the statistics of the generated Monte Carlo sample. Whereas $\left\langle M_{\mathrm{eff}}(P 520)\right\rangle$ is similar to the SM value, we have for $\mathrm{P} 600$ alone $\left\langle M_{\mathrm{eff}}(P 600)\right\rangle \simeq 1900 \mathrm{GeV}$. (As expected for supersymmetric signals, the tails are larger and the most probable value is not equal to the average effective mass.) The sum of Standard Model plus P600 weighted by the expected cross sections after cuts gives $\left\langle M_{\text {eff }}(S M+P 600)\right\rangle \simeq 1830 \mathrm{GeV}$. Thus for a point where the ratio of signal cross section to the Standard Model background is more than 3, additional information is indeed present in the effective mass at the level of two standard deviations, including the error on the Monte Carlo statistics. For P800 alone we have $\left\langle M_{\text {eff }}(P 800)\right\rangle \simeq 2230 \mathrm{GeV}$, hence $\left\langle M_{\mathrm{eff}}(S M+P 800)\right\rangle \simeq 1900 \mathrm{GeV}$.

We thus observe that even as the NMSSM signal decreases for the heavier scenarios, the reduction of the cross section is compensated in the distribution of the effective mass 


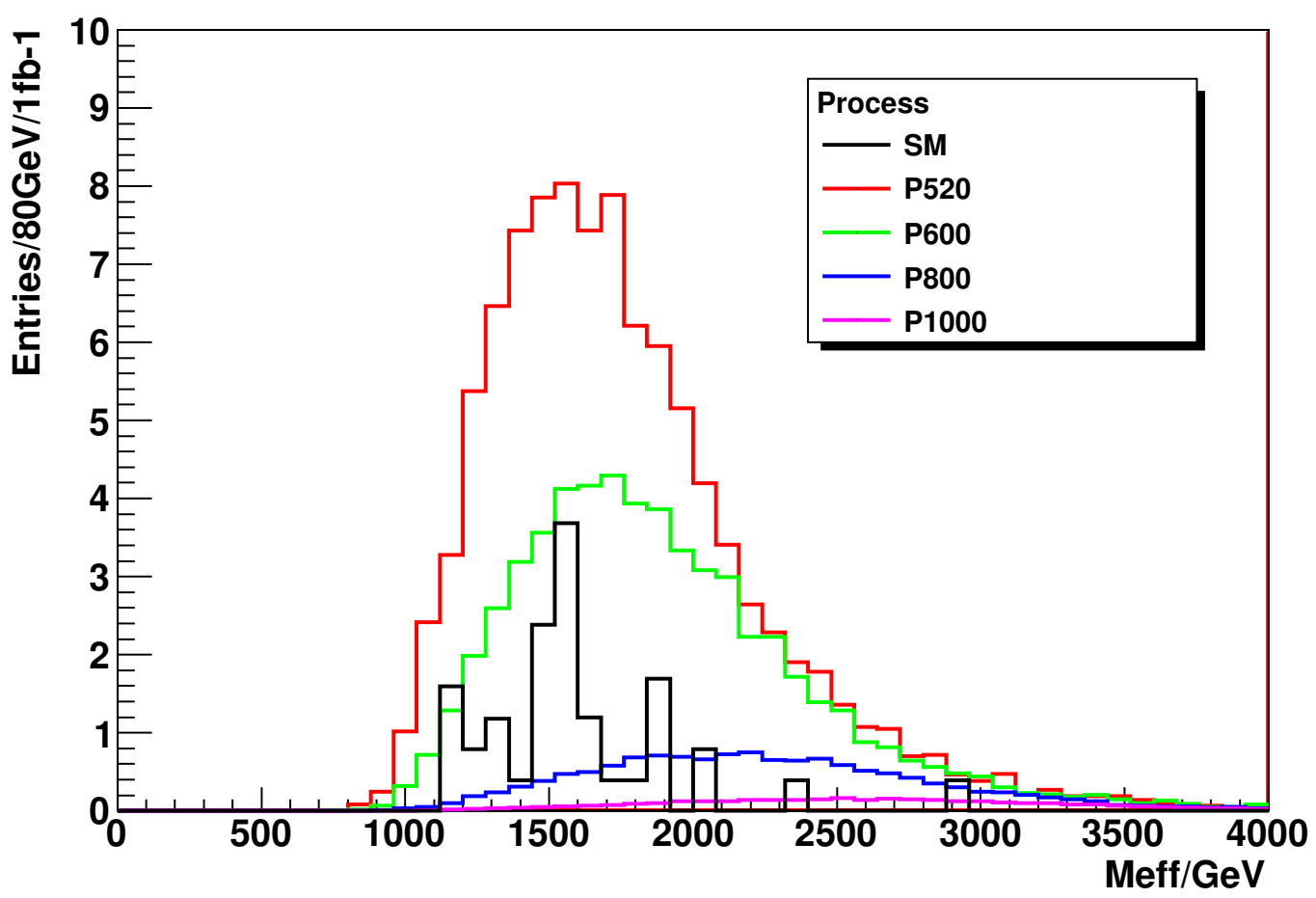

Figure 1. The effective mass distribution for the SM background and the cNMSSM points from table 1, after all cuts of the cNMSSM analysis are applied, normalised to an integrated luminosity of $1 \mathrm{fb}^{-1}$.

which still increases, ensuring even for P800 a difference of at least 2.5 standard deviations in addition to the counting measurement alone (see $\mathrm{S} / \sqrt{\mathrm{B}}$ in table 3 ). Obviously the study of $\left\langle M_{\text {eff }}\right\rangle$ gives further useful information.

For completeness we show in figure 2 the transverse momentum of the leading tau candidate (after the cNMSSM cuts). Modulo the rate, the spectrum of the leading tau candidate is slightly harder for the points with heavier squarks/gluinos.

\section{Comparison with the cMSSM in the stau coannihilation region}

It is well-known that $\tau$-rich final states from squark or gluino production would also be generated in the so-called stau coannihilation region of the (c)MSSM [29-33]. Hence the question arises, by which signatures this region of the cMSSM can be distinguished from the cNMSSM.

Clearly, the neutralino/ $\widetilde{\tau}$ spectrum of the cMSSM is different from the one of the cNMSSM: in the cMSSM (in the stau coannihilation region, which we assume henceforth) the lighter $\widetilde{\tau}_{1}$ has a mass close to the bino-like neutralino LSP $\chi_{1}^{0}$, whereas the neutralino $\chi_{2}^{0}$ is typically wino-like. At first sight, a squark decay cascade as in eq. (2.3) is also possible within the cMSSM, with corresponding replacements of the neutralinos $\chi_{1}^{0}$ and $\chi_{2}^{0}$. However, all right-handed squarks (and sleptons) would not couple to the wino-like $\chi_{2}^{0}$, and prefer to decay directly into the bino-like $\chi_{1}^{0}$. These decays do not lead to two $\tau$-leptons in the cascade. As a consequence, the $\tau$-rich cascades hardly occur for right-handed squark 


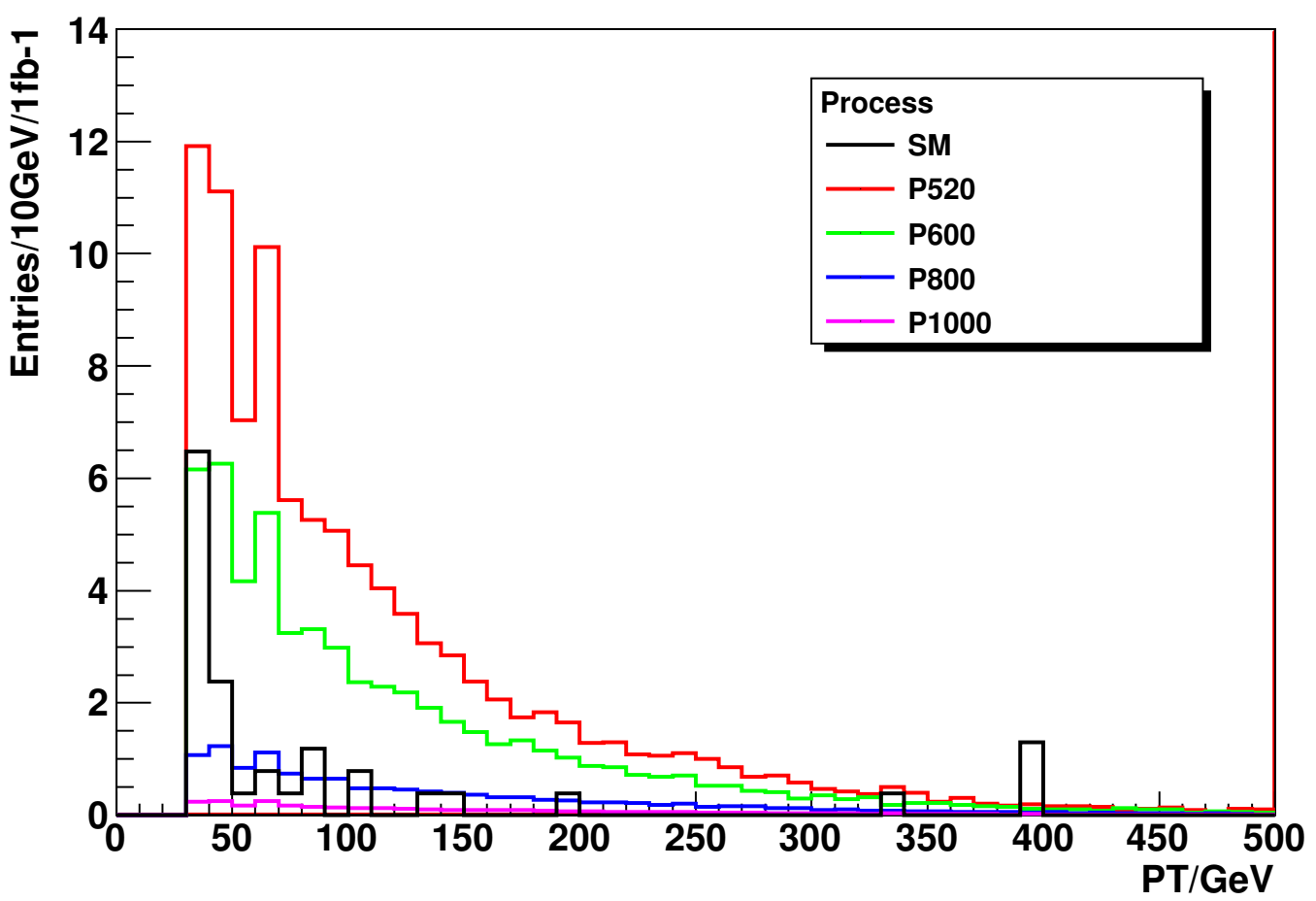

Figure 2. The transverse momentum distribution of the leading tau after all cuts of the cNMSSM analysis are applied, normalised to an integrated luminosity of $1 \mathrm{fb}^{-1}$.

decays, and are thus less frequent (relative to the total squark production cross section) than in the cNMSSM.

On the other hand, squarks and gluinos can be considerably lighter in the cMSSM than in the cNMSSM, since smaller values of $M_{1 / 2}$ - together with larger non-zero values of $m_{0}$ - are allowed. In particular this is the case, if we look for a point in the cMSSM parameter space with similar $\chi_{1}^{0}$ and $\widetilde{\tau}_{1}$ masses as the point P520 of the cNMSSM. It turns out that, for the corresponding values of $M_{1 / 2}$ and $m_{0}$ (we take $A_{0}=0$ for simplicity), the squarks and gluinos are considerably lighter than for the point P520. Hence we denote this point as MSSMl ("l" for "light"). Its parameters and sparticle masses are given in table 4 below.

As indicated in the last line in table 4, the lighter squarks and gluinos imply considerably larger production cross sections for the point MSSMl compared to P520. As a consequence, the number of events passing our cNMSSM analysis above is larger than for P520, in spite of the absence of $\tau$-leptons in right-handed squark decays.

There exist also points in the cMSSM parameter space where the squark and gluino spectrum resembles the one of P520, implying similar production cross sections. Such points correspond to larger values of $M_{1 / 2}$ and $m_{0}$; an example is given by the point MSSMh ("h" for "heavy"), whose squark and gluino masses are similar to those of the cNMSSM point P520 (see table 4).

The signal rates after the cNMSSM cuts for the points MSSMl and MSSMh are given in table 5: these are considerably larger (as compared to P520) for the point MSSMl, but 


\begin{tabular}{|l|c|c|}
\hline & MSSMl & MSSMh \\
\hline$M_{1 / 2}(\mathrm{GeV})$ & 360 & 520 \\
\hline$m_{0}(\mathrm{GeV})$ & 210 & 200 \\
\hline$A_{0}(\mathrm{GeV})$ & 0 & 0 \\
\hline $\tan \beta$ & 40 & 30 \\
\hline$\mu_{\text {eff }}(\mathrm{GeV})$ & 466 & 649 \\
\hline$m_{h_{1}^{0}}(\mathrm{GeV})$ & 114 & 117 \\
\hline$m_{\chi_{1}^{0}}(\mathrm{GeV})$ & 146 & 215 \\
\hline$m_{\chi_{2}^{0}}(\mathrm{GeV})$ & 274 & 406 \\
\hline$m_{\chi_{3,4}^{0}}(\mathrm{GeV})$ & 480 & 660 \\
\hline$m_{\chi_{1}^{ \pm}}(\mathrm{GeV})$ & 274 & 406 \\
\hline$m_{\chi_{2}^{ \pm}}(\mathrm{GeV})$ & 486 & 666 \\
\hline$m_{\tilde{g}}(\mathrm{GeV})$ & 851 & 1191 \\
\hline$m_{\tilde{u}_{L}}(\mathrm{GeV})$ & 800 & 1100 \\
\hline$m_{\tilde{u}_{R}}(\mathrm{GeV})$ & 776 & 1062 \\
\hline$m_{\tilde{d}_{L}}(\mathrm{GeV})$ & 804 & 1103 \\
\hline$m_{\tilde{d}_{R}}(\mathrm{GeV})$ & 774 & 1058 \\
\hline$m_{\tilde{t}_{1}}(\mathrm{GeV})$ & 598 & 843 \\
\hline$m_{\tilde{t}_{2}}(\mathrm{GeV})$ & 765 & 1038 \\
\hline$m_{\tilde{b}_{1}}(\mathrm{GeV})$ & 688 & 984 \\
\hline$m_{\tilde{b}_{2}}(\mathrm{GeV})$ & 749 & 1033 \\
\hline$m_{\tilde{e}_{L}}(\mathrm{GeV})$ & 322 & 401 \\
\hline$m_{\tilde{e}_{R}}(\mathrm{GeV})$ & 252 & 280 \\
\hline$m_{\tilde{\nu}_{l}}(\mathrm{GeV})$ & 312 & 393 \\
\hline$m_{\tilde{\tau}_{1}}(\mathrm{GeV})$ & 156 & 222 \\
\hline$m_{\tilde{\tau}_{2}}(\mathrm{GeV})$ & 332 & 405 \\
\hline$m_{\tilde{\nu}_{\tau}}(\mathrm{GeV})$ & 294 & 382 \\
\hline$\sigma(\mathrm{pb})$ & 9.44 & 1.40 \\
\hline & & \\
\hline
\end{tabular}

Table 4. SUSY breaking parameters, $\tan \beta, \mu_{\text {eff }}$, sparticle spectra and total sparticle cross sections for the cMSSM points MSSMl and MSSMh.

smaller for the point MSSMh in spite of the similar squark/gluino masses and hence the similar total sparticle cross section (see table 4). The reason was mentioned above: righthanded squarks do not decay via $\tau$-rich cascades and, hence, right-handed squark decays do not contribute to the signal after the cNMSSM analysis.

The $M_{\text {eff }}$ spectrum for the MSSM points, together with P520, P600 and the SM background, is shown in figure 3. First, the point MSSMl (with similar $\chi_{1}^{0}$ and $\widetilde{\tau}_{1}$ masses as the point P520) has not only a larger signal cross section as compared to P520, but we see that its maximum of $M_{\text {eff }}$ is visibly shifted towards smaller values.

Can we distinguish the point MSSMh from any of the cNMSSM points? Due to the similar squark/gluino masses as P520, MSSMh has its maximum of $M_{\text {eff }}$ in the same region 


\begin{tabular}{|c|c|c|c|c|}
\hline & & $4 \mathrm{j} 0 \mathrm{l}$ & $4 \mathrm{jtau}$ & cNMSSM analysis \\
\hline $\mathrm{MSSMl}$ & $9.4 \mathrm{pb}$ & $1429 \pm 10 \mathrm{fb}$ & $121 \pm 3 \mathrm{fb}$ & $166 \pm 4 \mathrm{fb}$ \\
$\mathrm{S} / \mathrm{B}$ & & 2.4 & 2.0 & 10 \\
$\mathrm{~S} / \sqrt{\mathrm{B}}$ & $1 \mathrm{fb}^{-1}$ & 58 & 15 & 42 \\
$\mathrm{~S} / \sqrt{\mathrm{B}}$ & $30 \mathrm{fb}^{-1}$ & 320 & 84 & 227 \\
\hline $\mathrm{MSSMh}$ & $1.40 \mathrm{pb}$ & $242 \pm 1.7 \mathrm{fb}$ & $22 \pm 0.5 \mathrm{fb}$ & $41 \pm 0.7 \mathrm{fb}$ \\
$\mathrm{S} / \mathrm{B}$ & & 0.4 & 0.46 & 2.5 \\
$\mathrm{~S} / \sqrt{\mathrm{B}}$ & $1 \mathrm{fb}^{-1}$ & 9.8 & 2.8 & 10 \\
$\mathrm{~S} / \sqrt{\mathrm{B}}$ & $30 \mathrm{fb}^{-1}$ & 54 & 15 & 56 \\
\hline
\end{tabular}

Table 5. Signal expectation for the MSSM points at NLO after all cuts. 120000 events per point minimum were generated. The error is the statistical error.

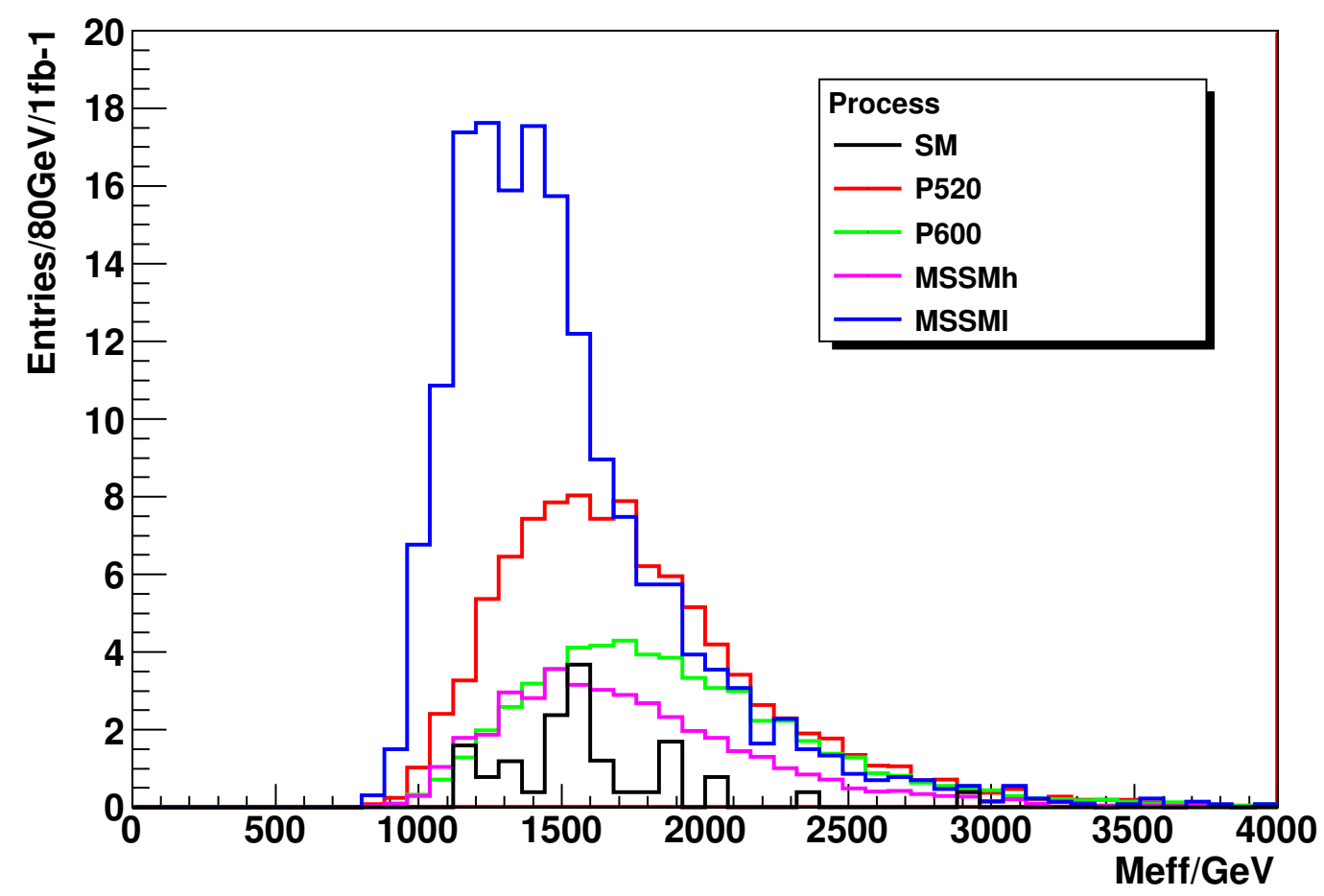

Figure 3. The effective mass distribution for the points P520, P600, MSSMl and MSSMh after all cuts of the cNMSSM analysis are applied.

as P520, but a significantly smaller signal rate. The signal rate for the cNMSSM point P600 of about $58 \mathrm{fb}$ is still larger than $41 \mathrm{fb}$ for MSSMh. (The difference is slightly larger than a conservative error of $20-30 \%$ on the theoretical cross section prediction.) On the other hand we see in figure 3 that the maximum of $M_{\text {eff }}$ for P600 is shifted towards larger values due to the heavier squarks/gluinos: the root mean square of the distributions is about $500 \mathrm{GeV}$ and the difference of the average effective mass is about $130 \mathrm{GeV}$, so that the error on the average effective mass for $1 \mathrm{fb}^{-1}$ is about $70 \mathrm{GeV}$, i.e., about two times smaller than the difference. The average effective mass is affected somewhat by the tails for large effective mass. Using a simple fit of the distributions, the peak to peak difference increases slightly 
to about $150 \mathrm{GeV}$ providing for a stronger separation of MSSMh and P600. Any cNMSSM point with still heavier squarks/gluinos (such that the signal rate coincides with the one for MSSMh) will imply a maximum for still larger values of $M_{\text {eff }}$. Hence, the cNMSSM points have either measurably larger signal rates after applying the cNMSSM cuts (if the maxima of $M_{\text {eff }}$ coincide with a MSSM point), or maxima at measurably larger values of $M_{\text {eff }}$ (if the signal rates coincide with a MSSM point).

Additionally one can compare the cross section ratios after the generic supersymmetric 4j0l cut, which are $242 \mathrm{fb}$ (MSSMh, where squarks are somewhat lighter than gluinos) as compared to $47 \mathrm{fb}$ (P600, where gluinos are somewhat heavier than squarks). Hence, given a corresponding signal in the data, a careful comparison of both the signal rates for generic and dedicated searches and the maximum of $M_{\text {eff }}$ (possibly including in addition the transverse momentum of the tau lepton) should allow to distinguish the cNMSSM from the MSSM in the stau coannihilation region.

\section{Conclusions and outlook}

In the present paper we have proposed criteria for the search for the fully constrained NMSSM at the LHC. In view of the relatively heavy squarks and gluinos in the cNMSSM and correspondingly small production cross sections, this task is not quite trivial. On the other hand, due to the large number of $\tau$-leptons in the final states, signatures involving hadronic $\tau$ decays are relatively efficient. Whereas the soft $\tau$-leptons in the final states are difficult to use, the requirement of at least one hard $\tau$-lepton has a relatively large signal acceptance.

Combining this requirement with relatively hard cuts on the transverse momenta of two jets and $E_{\mathrm{T}}^{\text {miss }}$ as specified at the end of section 3 , the signal to background ratio is significantly improved with respect to the more standard $4 \mathrm{j} 0 \mathrm{l}$ or $4 \mathrm{jtau}$ analyses. This result was obtained after simulations including detector effects and a $\tau$ acceptance, which we compared with and checked against the analysis of SUSY signals by the ATLAS group. Hence, already an integrated luminosity of $1 \mathrm{fb}^{-1}$ (at $14 \mathrm{TeV}$ c.m. energy) becomes sensitive to part of the parameter space of the cNMSSM whereas, trivially, more luminosity is required in case of heavier squarks and gluinos. In any case we believe that the cuts proposed here are the most sensitive ones to the parameter space of the cNMSSM. In addition we have discussed in how far a refined analysis employing both the signal rate and the maximum of $M_{\text {eff }}$ allows to distinguish the cNMSSM from the MSSM in the stau coannihilation region.

In the near future the LHC is on track to accumulate an integrated luminosity of $1 \mathrm{fb}^{-1}$ at $7 \mathrm{TeV}$ c.m. energy at the end of 2011 . We have estimated the number of signal events for the point P520, if we lower the cNMSSM cuts correspondingly: for two jets we require $p_{\mathrm{T}}>50$ and $20 \mathrm{GeV}$, respectively, and $E_{\mathrm{T}}^{\mathrm{miss}}>200 \mathrm{GeV}$. We obtain about 5 signal events passing these cuts.

For our analysis of signals of the cNMSSM at $14 \mathrm{TeV}$ c.m. energy we have left aside the presence of two soft $\tau$ leptons per event which represent, in principle, a spectacular signature for this class of models. In some regions of the parameter space of the cNMSSM 
— for very small values of $\lambda$ and/or a small $\widetilde{\tau}_{1}-\chi_{1}^{0}$ mass difference — the life time of $\widetilde{\tau}_{1}$ can be very small leading to displaced vertices of the decay $\widetilde{\tau}_{1} \rightarrow \tau+\chi_{1}^{0}$ into these soft $\tau$-leptons. Using dedicated track-based algorithms, the search for these soft $\tau$-leptons originating from displaced vertices is perhaps not completely hopeless.

In the framework of the general NMSSM, a singlino-like LSP can be accompagnied by a NLSP different from $\widetilde{\tau}_{1}$. The signatures of these scenarios would be very different from the ones discussed here (depending on the nature of the NLSP), and should also be investigated in the future.

\section{Acknowledgments}

We would like to thank T. Plehn and P. Wienemann for numerous discussions, K. Mawatari for help in interfacing PYTHIA with TAUOLA for supersymmetric models, and Sebastien Binet (LAL) for his invaluable help in automizing the production of the Alpgen samples. U.E. wishes to thank the Institut für Theoretische Physik in Heidelberg, where this work was started, for hospitality.

Open Access. This article is distributed under the terms of the Creative Commons Attribution Noncommercial License which permits any noncommercial use, distribution, and reproduction in any medium, provided the original author(s) and source are credited.

\section{References}

[1] M. Maniatis, The Next-to-Minimal Supersymmetric extension of the Standard Model reviewed, Int. J. Mod. Phys. A 25 (2010) 3505 [arXiv:0906. 0777] [SPIRES].

[2] U. Ellwanger, C. Hugonie and A.M. Teixeira, The Next-to-Minimal Supersymmetric Standard Model, Phys. Rept. 496 (2010) 1 [arXiv:0910.1785] [SPIRES].

[3] J.E. Kim and H.P. Nilles, The mu Problem and the Strong CP Problem, Phys. Lett. B 138 (1984) 150 [SPIRES].

[4] S.A. Abel, S. Sarkar and I.B. Whittingham, Neutralino dark matter in a class of unified theories, Nucl. Phys. B 392 (1993) 83 [hep-ph/9209292] [SPIRES].

[5] U. Ellwanger and C. Hugonie, Neutralino cascades in the $(M+1) S S M$, Eur. Phys. J. C 5 (1998) 723 [hep-ph/9712300] [SPIRES].

[6] U. Ellwanger and C. Hugonie, Topologies of the $(M+1) S S M$ with a singlino LSP at LEP2, Eur. Phys. J. C 13 (2000) 681 [hep-ph/9812427] [SPIRES].

[7] S. Hesselbach, F. Franke and H. Fraas, Displaced vertices in extended supersymmetric models, Phys. Lett. B 492 (2000) 140 [hep-ph/0007310] [SPIRES].

[8] V. Barger, P. Langacker and G. Shaughnessy, Neutralino signatures of the singlet extended MSSM, Phys. Lett. B 644 (2007) 361 [hep-ph/0609068] [SPIRES].

[9] G. Bélanger, F. Boudjema, C. Hugonie, A. Pukhov and A. Semenov, Relic density of dark matter in the NMSSM, JCAP 09 (2005) 001 [hep-ph/0505142] [SPIRES].

[10] J.F. Gunion, D. Hooper and B. McElrath, Light neutralino dark matter in the NMSSM, Phys. Rev. D 73 (2006) 015011 [hep-ph/0509024] [SPIRES]. 
[11] D.G. Cerdeno, E. Gabrielli, D.E. Lopez-Fogliani, C. Muñoz and A.M. Teixeira, Phenomenological viability of neutralino dark matter in the NMSSM, JCAP 06 (2007) 008 [hep-ph/0701271] [SPIRES].

[12] C. Hugonie, G. Bélanger and A. Pukhov, Dark Matter in the Constrained NMSSM, JCAP 11 (2007) 009 [arXiv:0707.0628] [SPIRES].

[13] G. Bélanger, C. Hugonie and A. Pukhov, Precision measurements, dark matter direct detection and LHC Higgs searches in a constrained NMSSM, JCAP 01 (2009) 023 [arXiv:0811.3224] [SPIRES].

[14] WMAP collaboration, E. Komatsu et al., Five-Year Wilkinson Microwave Anisotropy Probe (WMAP) Observations: Cosmological Interpretation, Astrophys. J. Suppl. 180 (2009) 330 [arXiv:0803.0547] [SPIRES].

[15] J.R. Ellis, J.F. Gunion, H.E. Haber, L. Roszkowski and F. Zwirner, Higgs Bosons in a Nonminimal Supersymmetric Model, Phys. Rev. D 39 (1989) 844 [SPIRES].

[16] M. Drees, Supersymmetric Models with Extended Higgs Sector, Int. J. Mod. Phys. A 4 (1989) 3635 [SPIRES].

[17] U. Ellwanger, M. Rausch de Traubenberg and C.A. Savoy, Particle spectrum in supersymmetric models with a gauge singlet, Phys. Lett. B 315 (1993) 331 [hep-ph/9307322] [SPIRES].

[18] T. Elliott, S.F. King and P.L. White, Unification constraints in the next-to-minimal supersymmetric standard model, Phys. Lett. B 351 (1995) 213 [hep-ph/9406303] [SPIRES].

[19] S.F. King and P.L. White, Resolving the constrained minimal and next-to-minimal supersymmetric standard models, Phys. Rev. D 52 (1995) 4183 [hep-ph/9505326] [SPIRES].

[20] U. Ellwanger, M. Rausch de Traubenberg and C.A. Savoy, Phenomenology of supersymmetric models with a singlet, Nucl. Phys. B 492 (1997) 21 [hep-ph/9611251] [SPIRES].

[21] A. Djouadi, U. Ellwanger and A.M. Teixeira, The constrained next-to-minimal supersymmetric standard model, Phys. Rev. Lett. 101 (2008) 101802 [arXiv:0803.0253] [SPIRES].

[22] A. Djouadi, U. Ellwanger and A.M. Teixeira, Phenomenology of the constrained NMSSM, JHEP 04 (2009) 031 [arXiv:0811.2699] [SPIRES].

[23] U. Ellwanger and C. Hugonie, NMSPEC: A Fortran code for the sparticle and Higgs masses in the NMSSM with GUT scale boundary conditions, Comput. Phys. Commun. 177 (2007) 399 [hep-ph/0612134] [SPIRES].

[24] A. Djouadi et al., Benchmark scenarios for the NMSSM, JHEP 07 (2008) 002 [arXiv:0801.4321] [SPIRES].

[25] C. Balázs and D. Carter, Discovery potential of the next-to-minimal supergravity motivated model, Phys. Rev. D 78 (2008) 055001 [arXiv:0808.0770] [SPIRES].

[26] D.J.H. Chung et al., The soft supersymmetry-breaking Lagrangian: Theory and applications, Phys. Rept. 407 (2005) 1 [hep-ph/0312378] [SPIRES].

[27] D.E. Morrissey, T. Plehn and T.M.P. Tait, Physics searches at the LHC, arXiv:0912.3259 [SPIRES].

[28] P. Nath et al., The Hunt for New Physics at the Large Hadron Collider, Nucl. Phys. Proc. Suppl. 200-202 (2010) 185 [arXiv:1001.2693] [SPIRES].

[29] J.R. Ellis, T. Falk and K.A. Olive, Neutralino-Stau Coannihilation and the Cosmological Upper Limit on the Mass of the Lightest Supersymmetric Particle, Phys. Lett. B 444 (1998) 367 [hep-ph/9810360] [SPIRES]. 
[30] J.A. Aguilar-Saavedra et al., Supersymmetry parameter analysis: SPA convention and project, Eur. Phys. J. C 46 (2006) 43 [hep-ph/0511344] [SPIRES].

[31] R.L. Arnowitt, B. Dutta, T. Kamon, N. Kolev and D.A. Toback, Detection of SUSY in the stau-neutralino coannihilation region at the LHC, Phys. Lett. B 639 (2006) 46 [hep-ph/0603128] [SPIRES].

[32] U. Chattopadhyay, D. Das, A. Datta and S. Poddar, Non-zero trilinear parameter in the mSUGRA model - dark matter and collider signals at Tevatron and LHC, Phys. Rev. D 76 (2007) 055008 [arXiv:0705.0921] [SPIRES].

[33] R.L. Arnowitt et al., Determining the Dark Matter Relic Density in the mSUGRA Stau-Neutralino Co-Annhiliation Region at the LHC, Phys. Rev. Lett. 100 (2008) 231802 [arXiv:0802.2968] [SPIRES].

[34] ALEPH collaboration, S. Schael et al., Search for neutral MSSM Higgs bosons at LEP, Eur. Phys. J. C 47 (2006) 547 [hep-ex/0602042] [SPIRES].

[35] U. Ellwanger, J.F. Gunion and C. Hugonie, NMHDECAY: A Fortran code for the Higgs masses, couplings and decay widths in the NMSSM, JHEP 02 (2005) 066 [hep-ph/0406215] [SPIRES].

[36] U. Ellwanger and C. Hugonie, NMHDECAY 2.0: An Updated program for sparticle masses, Higgs masses, couplings and decay widths in the NMSSM, Comput. Phys. Commun. 175 (2006) 290 [hep-ph/0508022] [SPIRES].

[37] G. Degrassi and P. Slavich, On the radiative corrections to the neutral Higgs boson masses in the NMSSM, Nucl. Phys. B 825 (2010) 119 [arXiv:0907.4682] [SPIRES].

[38] A. Djouadi, M.M. Muhlleitner and M. Spira, Decays of Supersymmetric Particles: the program SUSY-HIT (SUspect-SdecaY-HDECAY-InTerface), Acta Phys. Polon. B 38 (2007) 635 [hep-ph/0609292] [SPIRES].

[39] W. Beenakker, R. Hopker and M. Spira, PROSPINO: A program for the PROduction of Supersymmetric Particles In Next-to-leading Order QCD, hep-ph/9611232 [SPIRES] for updates see http://www.thphys.uni-heidelberg.de/ plehn/prospino/.

[40] The ATLAS collaboration, G. Aad et al., Expected Performance of the ATLAS Experiment - Detector, Trigger and Physics, arXiv:0901.0512 [SPIRES].

[41] T. Sjöstrand, S. Mrenna and P.Z. Skands, PYTHIA 6.4 Physics and Manual, JHEP 05 (2006) 026 [hep-ph/0603175] [SPIRES].

[42] M.L. Mangano, M. Moretti, F. Piccinini, R. Pittau and A.D. Polosa, ALPGEN, a generator for hard multiparton processes in hadronic collisions, JHEP 07 (2003) 001 [hep-ph/0206293] [SPIRES].

[43] S. Jadach, J.H. Kuhn and Z. Was, TAUOLA: A Library of Monte Carlo programs to simulate decays of polarized tau leptons, Comput. Phys. Commun. 64 (1990) 275 [SPIRES].

[44] M. Jezabek, Z. Was, S. Jadach and J.H. Kuhn, The tau decay library TAUOLA, update with exact $O(\alpha)$ QED corrections in $\tau \rightarrow \mu(e)$ neutrino anti-neutrino decay modes, Comput. Phys. Commun. 70 (1992) 69 [SPIRES].

[45] S. Jadach, Z. Was, R. Decker and J.H. Kuhn, The $\tau$ decay library TAUOLA: Version 2.4, Comput. Phys. Commun. 76 (1993) 361 [SPIRES].

[46] E. Richter-Was, AcerDET: A particle level fast simulation and reconstruction package for phenomenological studies on high $p_{T}$ physics at LHC, hep-ph/0207355 [SPIRES]. 